See discussions, stats, and author profiles for this publication at: https://www.researchgate.net/publication/24169601

\title{
The Universe in a Soap Film
}

ARTICLE in CLASSICAL AND QUANTUM GRAVITY · APRIL 2009

Impact Factor: $3.17 \cdot$ DOI: 10.1088/0264-9381/26/13/135018 · Source: arXiv

CITATIONS

2

\section{AUTHORS:}

Rohit Katti

Indian Institute of Science

2 PUBLICATIONS 4 CITATIONS

SEE PROFILE

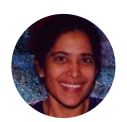

Supurna Sinha

Raman Research Institute

39 PUBLICATIONS 303 CITATIONS

SEE PROFILE
READS

25

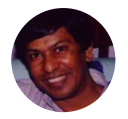

Joseph Samuel

Raman Research Institute

81 PUBLICATIONS $\quad 1,689$ CITATIONS

SEE PROFILE 


\title{
The Universe in a Soap Film
}

\author{
Rohit Katti, ${ }^{1,2}$ Joseph Samuel, ${ }^{1}$ and Supurna Sinha ${ }^{1}$ \\ ${ }^{1}$ Raman Research Institute, Bangalore, India 560080 \\ ${ }^{2}$ Department of Physics, Bangalore University, Bangalore, India 560056
}

\begin{abstract}
The value of the cosmological constant is one of the major puzzles of modern cosmology: it is tiny but nonzero. Sorkin predicted, from the Causet approach to quantum gravity, that the cosmological constant has quantum fluctuations. The predicted order of magnitude of the fluctuations agrees with the subsequently observed value of the cosmological constant. We had earlier developed an analogy between the cosmological constant of the Universe and the surface tension of fluid membranes. Here we demonstrate by computer simulations that the surface tension of a fluid membrane has statistical fluctuations stemming from its discrete molecular structure. Our analogy enables us to view these numerical experiments as probing a small and fluctuating cosmological constant. Deriving insights from our analogy, we show that a fluctuating cosmological constant is a generic feature of quantum gravity models and is far more general than the specific context in which it was originally proposed. We pursue and refine the idea of a fluctuating cosmological constant and work towards making further testable predictions.
\end{abstract}

PACS numbers: 04.60.-m, 82.39.Wj, 87.15.Kg, 87.16.Dg, 46.70.Hg, 68.03.-g 


\section{INTRODUCTION}

In the Sand Reckoner[4], Archimedes of Syracuse estimated the number of sand grains that would fill the then known Universe. Putting the spatial volume of the Universe at somewhat larger than our Solar system, he arrived at an astronomical number, which in modern notation is $10^{63}$. This was an early effort at grappling with the large numbers that are needed to describe our Universe. A modern version of the same endeavour would replace the spatial volume of the Universe by its spacetime four volume, which is around $10^{112} \mathrm{~cm}^{4}$. The modern analogue of "a grain of sand" is the smallest element of spacetime, which from our theories of relativity $(c)$, gravitation $(G)$ and quantum mechanics $(\hbar)$ is around the Planck length $\left(\hbar G / c^{3}\right)^{1 / 2}$ scale of $10^{-33} \mathrm{~cm}$. The known Universe is much larger now than in Archimedes' time and "the grains of sand" much finer. But the general idea is the same and we can estimate the number of "grains of sand" by dividing the four volume of the Universe by the Planck four volume. The answer turns out to be around $10^{244}$. We will refer to this as "Archimedes' Number" $\mathcal{N}_{\text {Arch }}$.

Dirac and large numbers: In a somewhat unusual paper, Dirac [10] put forward the hypothesis that large dimensionless numbers are unnatural in cosmology and that one should try to reduce the independent ones by finding relations between such numbers. In the last two decades, there have been systematic observations [9, 29, 31, 49] of dim and distant supernovae, which clearly indicate the presence of a tiny (in natural Planck units $\hbar=c=G=1$ ) but non zero cosmological constant $\Lambda \approx 10^{-122}$. Small dimensionless numbers in cosmology are as embarrassing as large ones, since the inverse of a small number is a large one. Can one relate these two large numbers $\mathcal{N}_{\text {Arch }}$ and $\Lambda^{-1}$ ? This was precisely what was done by Sorkin [38] in a remarkable prediction made before the supernova observations [9, 29, 31]. Sorkin argued that quantum gravity effects would predict an order of magnitude for fluctuations in the cosmological constant which in natural units is $1 / \sqrt{\mathcal{N}_{\text {Arch }}}$. This is precisely the order of magnitude of the observed value of the cosmological constant. Sorkin's proposal was made in the context of Causal Sets, which is one of several approaches to quantum gravity. We will argue here, using an analogy developed earlier, that Sorkin's proposal is far more general than the context in which it was proposed. A fluctuating $\Lambda$ is a generic feature of quantum theories of gravity and is one of the few predictions which have emerged from them. Understanding the fluctuations in more detail may lead to testable 
predictions which could be used to rule out candidate quantum theories of gravitation.

Approaches to Quantum Gravity: While there are many theoretical approaches to quantum gravity, each with its own adherents, few of these approaches have been developed to the point where they make significant contact with the real world in the form of testable experimental predictions. Some quantum gravity models violate Local Lorentz invariance and predict non Lorentz covariant dispersion relations for electromagnetic radiation propagating in vacuo. There have been proposals[3] to test such models using gamma ray burst observations. However, not all quantum gravity models, predict violation of Local Lorentz invariance. A more generic feature of quantum gravity models is an underlying discreteness of spacetime at the Planck scale. It is clear from simple dimensional arguments and thought experiments involving Heisenberg microscopes that spacetime events cannot be localised to better than Planck scale accuracy. We learn from semiclassical gravity that black hole entropy is finite and given by the number of Planck areas in the horizon. This is a persuasive argument that spacetime is discrete at the Planck scale. Some possible tests of this discreteness have been explored in [11, 30]. From this discreteness, we are naturally led to consider Archimedes' number $\mathcal{N}_{\text {Arch }}$ and look for possible ways to make predictions which can be tested.

Avogadro's Number and Brownian Motion: Let us now turn from large numbers in cosmology to large numbers in the laboratory. A familiar large number is Avogadro's number $\mathcal{N}_{\text {Avo }} \approx 10^{23}$ the number of atoms in a mole of chemical substance. Atoms are so small and there are so many of them, even in a grain of sand, that it is hard to discern the atomistic nature of matter in most experiments. This impasse was broken around a hundred years ago by Einstein's work on Brownian motion. It became possible to deduce the existence of atoms by observations of pollen grains using an optical microscope, a coarse instrument by atomic standards. Brownian motion is due to fluctuation effects resulting from the atomic structure of matter. Such effects are of order $1 / \sqrt{\mathcal{N}_{A v o}}$ which is not quite as small as $1 / \mathcal{N}_{\text {Avo }}$ and therefore of sensible magnitude. Could it be that the cosmic acceleration which has been revealed by supernova observations represents cosmic fluctuations caused by the otherwise unobservable, quantal (Planck scale) discreteness of spacetime? Could the accelerating Universe be a modern analogue of Brownian motion? These are the questions we address in this paper. We pursue Sorkin's idea and try to understand it from a general point of view. Our larger objective is to translate the idea into firm testable predictions 
and generate a confrontation between theory and observations.

Analogue Gravity: Condensed matter analogues of fundamental physics are a valuable aid to understanding [5, 44, 46]. Particle physics has imbibed ideas like spontaneous symmetry breaking from analogies with magnetic systems and superconductivity. Historically, the Higgs phenomenon of elementary particle physics was first noticed in superconductivity [26]. Such cross fertilization of ideas enriches both fundamental physics [12, 13] and its laboratory analogue. Analogue models for gravity are as old as GR itself[5]. Laboratory analogues [5, 42] of Hawking radiation have been discussed in the context of supersonic fluid flows and Bose Einstein condensates. Another example is the analogy [8, 45] between defects in liquid crystals and phase transitions in the early universe. Such analogies provide a concrete context for discussing fundamental physics and sometimes an experimental basis for the discussion. In some domains of fundamental physics, like gravity at the Planck scale, experiments cannot be performed because they are beyond our reach in energy. Laboratory analogues are therefore extremely valuable as they are the nearest one can get to experimental quantum gravity. They bring abstract ideas of fundamental physics down to earth and into the laboratory. While laboratory analogues of particle physics, classical and semiclassical gravity have been discussed earlier, laboratory analogues of quantum gravity effects are much rarer. Some of these [5] probe violations of local Lorentz invariance, which are present in some quantum gravity models. A more generic feature of quantum gravity models is discretess of spacetime in some form, which is present in all quantum gravity models. Volovik [46] has shown how the Universe can be seen in a Helium droplet. We show here how the Universe can be seen in a soap film. In this paper we pursue an analogy between quantum gravity and soft condensed matter. Soft matter systems are dominated by thermal rather than quantum fluctuations. Such systems can be studied by table top experiments that are relatively easy to perform.

Summary of paper: This article is organised as follows. We will first review the necessary background, describing the cosmological constant problem, Sorkin's proposed resolution of it and our previously developed analogy between surface tension and the cosmological constant. We then describe numerical experiments on fluid membranes that reveal a small and fluctuating surface tension. By analogy this amounts to probing a small and fluctuating cosmological constant. We then use the analogy to arrive at the conclusion that the fluctuating $\Lambda$ idea is a robust one and would be generically present in any approach to quantum gravity. We conclude with a discussion 
of the limitations of the analogy and some future directions.

\section{REVIEW OF PREVIOUS WORK}

The Cosmological Constant Problem: The cosmological constant has long been in disgrace because of its murky origins. Einstein introduced it into General Relativity for the wrong reasons, then faced with Hubble's observations, recanted and took it out (again for the wrong reasons). For much of the history of GR, the cosmological constant suffered benign neglect because of its tainted past. GR was successfully applied to solar system physics without a cosmological constant. In a seminal paper on the cosmological constant [33], Zeldovich let the genie out of the bottle and it has never successfully been put back since. From quantum field theory we know that all the fields in nature have vacuum "zero point" energy. If we sum over all the modes present in the theory we get a divergent answer, which has to be regulated by applying a cutoff at the Planck scale. This results in a cosmological constant of order one in Planck units. In fact, the observed value

of the cosmological constant is tiny but non zero $\Lambda l_{\text {Planck }}^{4} \approx 10^{-122}$. (Our choice of units for $\Lambda$ is nonstandard but convenient.) This results in the cosmological constant problem:

a) Why is the cosmological constant so small?

b) Why (if it is so small) does it exist at all?

This is the same problem that faced the learned men of Brobdingnag when they were confronted with Gulliver and asked to account for his small stature: why was he so small? And why (if he was so small) did he exist at all? It is not easy to solve this problem since the two parts of it are in opposition. If we succeed in explaining the smallness, we would be hard put to explain the existence (and vice versa). Explaining a small non zero cosmological constant brings us up against Dirac's large numbers again.

Sorkin's proposal: In 1990, Sorkin proposed using the Causet approach to quantum gravity that the smallness of $\Lambda$ could be understood as a quantum fluctuation. Sorkin's Causet approach to quantum gravity replaces spacetime by a discrete structure, a collection of points carrying causal relations. The number $\mathcal{N}_{\text {Arch }}$ of points is the four volume $\mathcal{V}$ of spacetime (more precisely the 
four volume of the causal past of a cosmic observer in Planck units). The rest of the metrical information in spacetime (the conformal structure) is captured in causal relations between points.

Sorkin's proposal addresses only part b) of the cosmological constant dilemma. Let us for the moment suppose that part a) has been solved: some mechanism has been found for ensuring that the expectation value $\langle\Lambda>$ of the cosmological constant is zero. Sorkin's idea is that there will be Poisson fluctuations $\Delta \Lambda$ about this mean value which result in a small nonzero cosmological constant $\Lambda=<\Lambda>+\Delta \Lambda=\Delta \Lambda$. From the uncertainty principle $\Delta \Lambda \Delta \mathcal{V} \approx 1$. From Poisson statistics, $\Delta \mathcal{N}_{\text {Arch }} \approx \sqrt{\mathcal{N}_{\text {Arch }}}$. These $\sqrt{\mathcal{N}_{\text {Arch }}}$ fluctuations are the mechanism for a small and nonzero cosmological constant. Based on this argument from quantum gravity, Sorkin predicts [38] the following order of magnitude for the fluctuations in $\Lambda$ :

$$
\Delta \Lambda \approx \frac{l_{\text {Planck }}^{-4}}{\sqrt{\mathcal{N}_{\text {Arch }}}}
$$

This gives a logical basis for relating $\Lambda^{-1}$ with the Archimedes' number $\mathcal{N}_{\text {Arch }}$ in accordance with Dirac's large number hypothesis.

Astronomical Evidence: Sorkin's prediction is also in accord with astronomical data. There is a growing body [9] of observational evidence (e.g redshift-luminosity distance relations from type I supernovae) which shows that the Universe is accelerating at the present epoch, indicative of a positive cosmological constant. The energy density in the cosmological constant (referred to as dark energy by astronomers) is comparable in magnitude to the energy density of matter in the Universe. Sorkin's argument predicts the correct order of magnitude for $\Lambda$ but makes no prediction about the expected sign of the cosmological constant. Neither is there any prediction from Causet theory that solves part a). It assumes that part a) is solved by some other mechanism and then supplies the small fluctuation which would solve part b) and explain the observed value of $\Lambda$. Other researchers [27, 47] have also followed on Sorkin's idea with slight variations.

Analogy: In [34] we developed an analogy between the Universe and a fluid membrane. The analogy is based on the usual mapping between quantum field theory and statistical mechanics. The geometric description of a membrane as a surface in space corresponds to the geometric description of spacetime as a four dimensional manifold. The quantum fluctuations of spacetime map to the thermal fluctuations of a membrane. The breakdown of the smooth geometric picture at the molecular scale $l_{\text {mol }}$ corresponds to the Planck scale discreteness of spacetime expected 


\section{Table of Analogy}

\begin{tabular}{ll}
\hline Membranes & Universe \\
Configuration $\mathcal{C}$ & History $\mathcal{H}$ \\
Area of a configuration & Four volume of a history \\
Sum over configurations & Sum over histories \\
Energy $\mathcal{E}(\mathcal{C})$ & Classical Action $\mathcal{I}(\mathcal{H})$ \\
$\mathcal{E}_{0}=a_{0} \int d^{2} x \sqrt{\gamma}$ & $I_{0}=c_{0} \int d^{4} x \sqrt{-g}$ \\
$\mathcal{E}_{2}=a_{2} \int d^{2} x \sqrt{\gamma} H^{2}$ & $I_{2}=c_{2} \int d^{4} x \sqrt{-g} R$ \\
Minimum energy configuration & Classical Path of Least Action \\
Temperature $T$ & Planck's constant $\hbar$ \\
Thermal Fluctuations & Quantum Fluctuations \\
Surface Tension $\sigma$ & Cosmological Constant $\Lambda$ \\
Free Energy & Effective Action \\
Molecular Length $l_{\text {mol }}=.3 \mathrm{~nm}$ & Planck Length $l_{P}=10^{-33} \mathrm{~cm}$ \\
Molecules & Causet elements \\
Molecular level discreteness of space & Planck scale level discreteness of space-time \\
\hline
\end{tabular}

TABLE I: Table shows the corresponding elements in the analogy between the Universe and Fluid Membranes

from quantum gravity. The analogy is explained in Ref. 34] to which the reader is referred for an explanation of notation and more details. For the reader's convenience, we have displayed the main points in tabular form for quick reference (table 1).

It is worth emphasizing that the analogy is of a formal and mathematical nature (rather than a physical one). As a result we have mappings between physically disparate elements. For example the analogue of the cosmological constant of the Universe is the surface tension of a membrane. The statics of membranes is mapped to the dynamics of spacetime. What is striking about our analogy is that it maps the exotic and ill understood physics of quantum gravity into relatively well understood physics, which is testable by laboratory and numerical experiments. 
To illustrate the analogy, we show how renormalisation effects generate a surface tension which is of order $\sigma_{0}=T / l_{m o l}^{2}$. Here $T$ is the temperature and $l_{m o l}$ a molecular length scale, which is around nanometres. We will sometimes use "natural units" and set $T$ and $l_{m o l}$ to unity. As an instructive example, we start with a microscopic energy in which the surface tension is set to zero by hand. Consider such a membrane whose equilibrium configuration is a plane rectangle with sides $L_{1}, L_{2}$ and area $\mathcal{A}=L_{1} L_{2}$. Due to thermal fluctuations, the membrane will vibrate about its equilibrium configuration. Assuming small vibrations, we can model them as harmonic oscillators and expect by equipartition that the expectation value of energy $\langle E\rangle$ in each mode is $T$. Performing a sum over modes to evaluate the contribution from all the modes we find a divergent answer which has to be regulated by the molecular scale cutoff.

$$
T \iint_{0}^{k_{\max }} \frac{d^{2} k d^{2} x}{(2 \pi)^{2}}
$$

where $\vec{k}$ is a wave vector and $k_{\max }=2 \pi / l_{\text {mol }}$ is the cutoff set by the molecular scale. Performing the $k$ integral we find that this contributes a term

$$
\frac{\pi T}{l_{m o l}^{2}} \int d^{2} x=\frac{\pi T}{l_{m o l}^{2}} \mathcal{A}
$$

to the energy, giving rise to a surface tension $\sigma_{0} \approx T / l_{m o l}^{2}$ of order 1 in dimensionless units. Even if one assumes that the microscopic energy has zero surface tension, such a term is generated by "radiative corrections" in a manner analogous to the generation of vacuum energy from the Casimir effect. So we would expect that most membranes have a value of order $\sigma_{0}$ for the surface tension. This expectation is borne out for most interfaces. However, there is a notable exception -fluid membranes- which we now turn to.

fluid membranes: Fluid membranes[32] are composed of amphiphilic molecules, which consist of hydrophilic (water loving) polar head groups and hydrophobic (water hating) hydrocarbon tails. Common examples of amphiphilic molecules are soaps and detergents, phospholipids, cholesterol and glycolipids. If one adds amphiphiles to water, they cluster together to hide their tails and so form supramolecular structures like micelles, vesicles and symmetric bilayers. Bilayers of phospholipids are studied by physicists as simple models for the biological cell membrane. These bilayers are sometimes called fluid membranes as the amphiphilic molecules flow freely on the two dimensional surface of the membrane. 
A fluid membrane, that is not subject to external constraints, naturally assumes a tensionless state. From dimensional arguments, we would have expected a surface tension of order $\sigma_{0}$. This works out to about 40 milliJoules $/ \mathrm{m}^{2}$ in conventional units. Indeed, most interfaces have a surface tension which is of this order. Fluid membranes are an exception in having vanishing surface tension. A tensionless fluid membrane solves part a) of the analogue cosmological constant problem. It was shown in [34] that the fluid membrane also solves part b) by virtue of its small surface tension fluctuations. This is a direct analog of Sorkin's suggestion [39] that there are small fluctuations in the cosmological constant due to spacetime discreteness on quantum gravity scales within the causet framework[7].

The Analogue Cosmological Constant Problem: From fairly simple theory [25, 32] one can understand the tensionless state of fluid membranes. As one increases the areal density of molecules in the fluid membrane, the area per molecule $\alpha$ decreases. There is a limit to this packing density however, and at a critical value of $\alpha=\alpha_{0}$, there is a minimum in the free energy per molecule $f(\alpha)$. A fluid membrane which can adjust its areal density does so to attain this critical value of $\alpha$ and is said to be saturated. At the saturation point $\alpha=\alpha_{0}$ the free energy per molecule has a minimum

$$
\left.\frac{\partial f}{\partial \alpha}\right|_{\alpha=\alpha_{0}}=0
$$

Consider a saturated membrane with area $\mathcal{A}$ and $N=\mathcal{A} / \alpha$ molecules. The total free energy is given by [25, 32] $F(\mathcal{A})=N f(\alpha)$. The expected value of the surface tension of the membrane vanishes:

$$
<\sigma>=\frac{\partial F}{\partial \mathcal{A}}=\left.\frac{\partial f}{\partial \alpha}\right|_{\alpha_{0}}=0
$$

From the analogy developed earlier [34], this corresponds to a vanishing cosmological constant and solves the first horn of the cosmological constant dilemma.

This simple theory also predicts that a fluid membrane consisting of $N$ molecules has an interfacial tension $\sigma$ which fluctuates about zero. The mean square statistical fluctuation in the surface tension is:

$$
(\Delta \sigma)^{2}=<(\sigma-<\sigma>)^{2}>=T \frac{\partial^{2} F}{\partial \mathcal{A}^{2}}=\left.\frac{T}{N} \frac{\partial^{2} f}{\partial \alpha^{2}}\right|_{\alpha_{0}} .
$$


On dimensional grounds, we can expect $\sigma_{0} \approx \sqrt{\left.T \frac{\partial^{2} f}{\partial \alpha^{2}}\right|_{\alpha_{0}}}$ and so

$$
\Delta \sigma \sim \frac{\sigma_{0}}{\sqrt{N}}
$$

in complete analogy to Sorkin's proposal in the cosmological context. From the simple theory described above we would expect that fluid membranes which can adjust their areal density (either by exchanging lipid molecules with the ambient solution or by adjusting their area) a) have vanishing surface tension and b) have surface tension fluctuations about zero with a magnitude $\sigma_{0} / \sqrt{N}$, where $N$ is the number of lipid molecules.

Experiments: What is the experimental situation? Fluid membranes have been extensively studied both theoretically and experimentally for many years (see Ref. [18] for some references). It has long been realised that fluid membranes can exist in a tensionless state. The effects of the vanishing surface tension can be seen in the variety of shapes assumed by red blood cells, which is well explained theoretically on the basis of a vanishing surface tension. The flickering of red blood cells due to thermal fluctuations has also been noted. However, quantitative experiments do not usually yield a value for this small tension but only place upper bounds. For instance, Kwok and Evans[23] probe the surface tension of a fluid membrane using pipette aspiration techniques. The upper bound they give is three orders of magnitude smaller than dimensionally expected $\sigma_{0}=40 \mathrm{~mJ} / \mathrm{m}^{2}$.

The fluctuating surface tension of part b) was first predicted in [34] and an experiment was proposed to measure it. The proposed experiment involved a cylindrical fluid membrane in an ambient solution stretched between two tiny rings, both of radius $r$ in tens of nanometers. One of the rings is attached to a piezoelectric translation stage which can be moved in nanometer steps. The other ring is attached to a micron sized bead which is confined in an optical trap. Fixing the separation $L$ by using a feedback loop, one can measure the force $F$ on the bead. This force $F$ is related to the surface tension by $F=2 \pi r \sigma$. We expect to see fluctuations [34, 37] in the surface tension due to finiteness of $N$ over and above any instrumental noise which may be present. Such an experiment can be viewed as an analog quantum gravity experiment probing a small nonzero fluctuating cosmological constant. There are practical, though not insurmountable, difficulties of working with real laboratory fluid membranes which are small enough to show an appreciable fluctuation. In contrast, a computer simulation is cleaner and the relevant tunable parameters are 
more in control. This motivated us to use a computer simulation or a "numerical experiment" to test our theoretical predictions.

\section{SIMULATIONS OF FLUID MEMBRANES}

In Molecular Dynamics Simulations (MDS) [14] one integrates the microscopic equations (Newton's equations) that govern the motion of each atom. Such simulations are computationally expensive and therefore not very efficient when one has to study the behaviour of a system containing a large number of particles for a long time. A more efficient scheme called Dissipative Particle Dynamics (DPD) has been developed[14, 19], which groups atoms into beads and uses a coarse grained description. The beads interact by conservative forces as well as dissipative and random forces. These last two are chosen, consistent with the Fluctuation-Dissipation Theorem, to ensure that the system equilibriates to a Boltzmann distribution. It has been shown [17] that DPD is orders of magnitude more efficient than MDS and gives the same results. The increased efficiency of DPD permits us to study the surface tension fluctuations of fluid membranes for a range of membrane sizes. The DPD programmes we used were provided to us by M. Venturoli. More details of these programmes are published in [22]. The programmes were intended to study biological membranes and have far more realistic detail and flexibility than we really need for our purposes. We expect that the broad conclusions of our study do not depend on these realistic details.

Simulation Model: The system is subject to periodic boundary conditions in the $x, y$ and $z$ directions so that the simulation box has the topology of a three-torus. The box has equal extent in the $x$ and $y$ directions and a larger extent (initially) in the $z$ direction. The DPD programme starts with an initially random distribution of lipid and water molecules. The initial configuration is evolved and the molecules spontaneously assemble into a bilayer lying in the $x-y$ plane. The topology of the membrane is a two torus and the geometry of its equilibrium configuration is flat intrinsically and extrinsically. The total number of lipid molecules (counting those on both sides of the symmetric lipid bilayer) is varied from 40 to 400. A snapshot of a bilayer with 400 molecules is shown in Fig.1. These numbers have been chosen optimally: for small numbers of lipid molecules, the bilayer becomes unstable and larger numbers are computationally expensive. Such mesoscale 


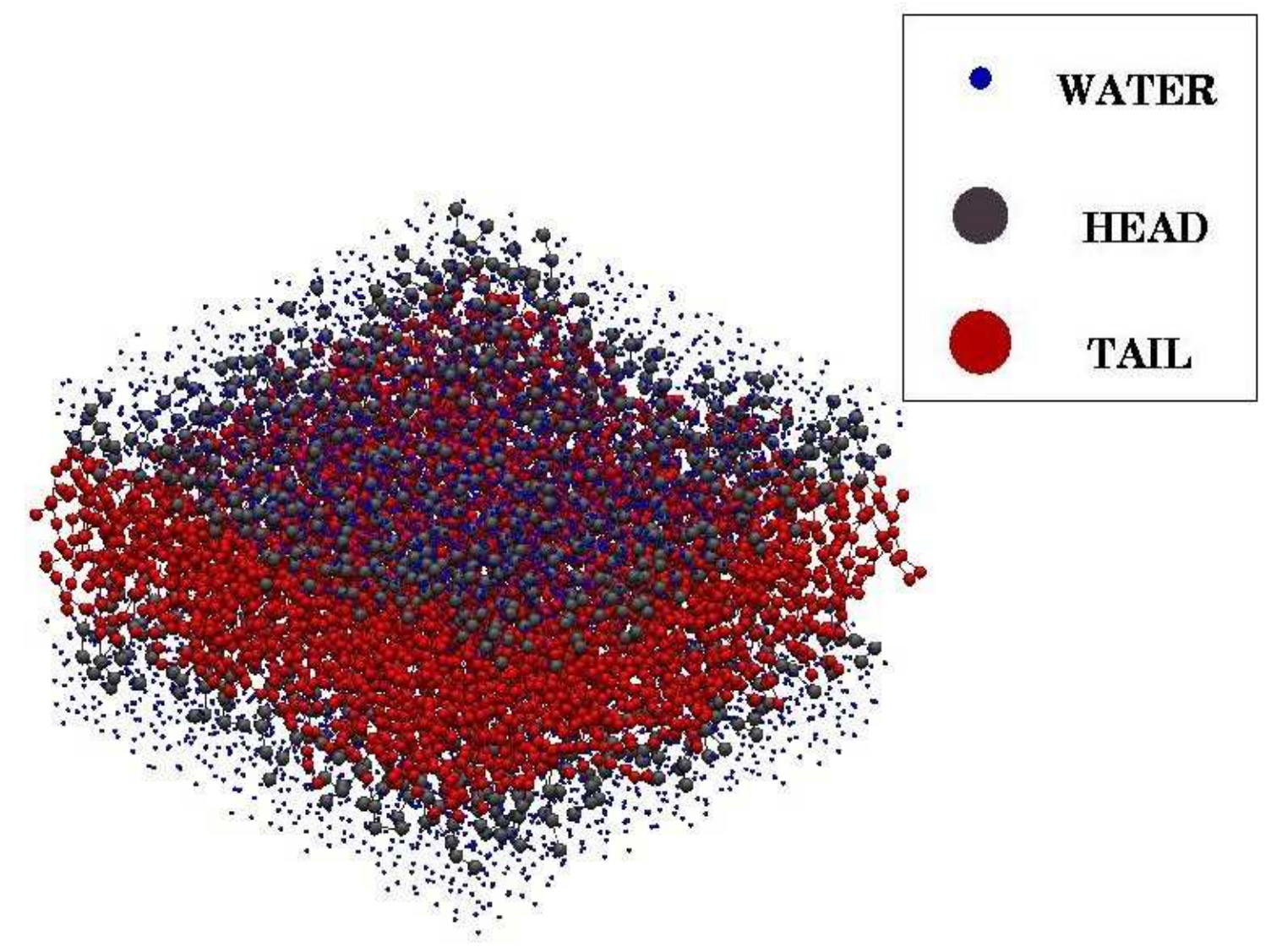

FIG. 1: The figure shows a system of 400 lipid molecules forming a symmetric bilayer with 200 molecules on each side. The system is subject to periodic boundary conditions in the $x-y-z$ directions.

membranes enable us to probe surface tension fluctuations.

The simulation model[22] uses three types of atoms $w, h$ and $t$ to represent the water, head and tail atoms of a lipid molecule respectively. The total force on a DPD particle $i$ is a sum over all the other particles $j$, of three pairwise additive type forces: conservative, dissipative and random. The conservative forces between the particles are all repulsive and given by:

$$
\begin{aligned}
F_{i j}^{C} & =a_{i j}\left(1-\left|\vec{r}_{i j}\right| / R_{c}\right) \hat{r}_{i j} \text { if }\left|\vec{r}_{i j}\right|<R_{c} \\
& =0 \quad \text { otherwise }
\end{aligned}
$$


$R_{c}$ is the range of the interaction and serves as our unit of length. This also determines a unit of time through the mean thermal velocity[22]. The coefficients $a_{i j}>0$ representing the repulsion strength are suitably chosen to mimic hydrophobic and hydrophilic interactions. We use $a=1$ as the unit of energy and the $a_{i j} \mathrm{~s}$ are chosen as $a_{w w}=a_{t t}=25, a_{w h}=15, a_{h h}=35$ and $a_{w t}=80$ following [22]. All numbers are expressed in in these units. The temperature $T$ was set to .7 in all the runs. The atoms in the lipid molecules are connected via harmonic springs with a spring constant $k_{r}=100$ and equilibrium distance $r_{0}=0.7$. The bond bending potential between two consecutive bonds is given in terms of a bending spring constant of $k_{\theta}=10$ and equilibrium angle $\theta_{0}=180^{\circ}$.

Calculating the Surface Tension: The surface tension of the membrane has been computed as follows. At each point of the membrane, the stress tensor is computed from the microscopic forces between pairs of atoms. There is a "gauge" ambiguity [35] in associating a stress field with a force between two atoms. This ambiguity arises because our description does not include a mechanism for interaction between atoms. (The van der Waal interaction is fundamentally electromagnetic and would entail distributed Maxwell stresses.) A reasonable and widespread choice [22, 35] is to suppose that the stress is concentrated along the shortest straight line joining each pair of atoms. After summing over all pairs, we arrive at the total stress tensor $\sigma_{i j}$.

From symmetry, the stress tensor is expected to have principal directions normal and tangential to the membrane. The normal component is $p_{N}(z)$ and the two lateral components are $p_{L}(z)$. Both these pressures have an intricate variation across the membrane. The surface tension was computed from the formula[22]:

$$
\sigma=\int d z\left(p_{N}(z)-p_{L}(z)\right)
$$

where the integral is over the thickness of the membrane. This integral was evaluated numerically and the result was averaged over 100 runs. Each run consisted of evolving the system for 10,000 timesteps (where each timestep translated into real units is of the order of 25 picoseconds). This amounts to time averaging the surface tension. We may believe from the ergodic theorem that this is equivalent to performing an ensemble average.

The formula (10) for the surface tension is free of the "gauge" ambiguities that appear in the stress field. It can be readily understood by considering a spherical membrane and computing the 
pressure difference between the inside and outside of the membrane by integrating the equilibrium condition $\nabla_{i} \sigma^{i j}=0$, for the stress tensor $\sigma^{i j}$ across the membrane, and using Laplace's law relating the surface tension to the pressure difference and the extrinsic curvature of the membrane.

A real bilayer membrane which is not subject to external constraints is expected to assume a tensionless state. However, bilayers assembled via simulations are constrained by the finite size of the simulation box and this induces lateral tensions on the membrane, so it takes some effort to attain a tensionless state[15, 36]. With $N$ fixed, we vary the lateral size of the simulation box and thus vary $\alpha$. In Fig. 2 we plot the membrane tension versus $\alpha$, the area per lipid for different numbers of molecules. From the simple theory explained above, we would expect that the surface tension $\sigma=f(\alpha)$ depends only on $\alpha$ and doesn't depend on $N$. This is precisely what was seen in the simulations. To a very good approximation, the graphs for different $N$ values fall on top of each other. This enables us to locate the area per molecule pertaining to the tensionless state. (We are being a bit sloppy here. The $\alpha$ plotted above is the projected area per lipid, which is equal to the area per lipid only in the equilibrium state.) We repeat this for a number of different values of total number of molecules. We notice that at a preferred value of the area per molecule $\alpha_{0}$ (around 1.57 in all cases) the membrane is tensionless. (See Fig. 2). This is entirely in agreement with the standard picture for tensionless membranes. If we allow the simulation box to adjust itself and "relax" under the pull of the lipids, the membrane naturally assumes a tensionless state. This is the analogue of the vanishing cosmological constant of the Universe and solves part a).

Regarding part b) (the fluctuating surface tension), in Fig. 3, we plot the standard deviation $\Delta \sigma$ of surface tension as a function of $N$, the number of lipids. The log-log plot clearly shows the $1 / \sqrt{N}$ dependence of the surface tension on $N$. As $N$ tends to infinity, we expect to find vanishing surface tension fluctuations in the thermodynamic limit. The coefficient of $1 / \sqrt{N}$ agrees in order of magnitude with $\sigma_{0}$ as expected. This numerical experiment can be viewed as a demonstration of Sorkin's idea of a small and fluctuating cosmological constant.

The fluctuation effects we see in the membrane simulations are entirely standard predictions from Statistical Mechanics [24]. Consider a system described by a set of variables $X$, whose entropy is given by $S(X)$. A thermodynamic system maximises its entropy subject to the constraints imposed on it. This determines the equilibrium value of $X$, which we call $X_{0}$. Since $X_{0}$ is a maximum of the entropy the first derivatives of $S(X)$ vanish at $X_{0}$. Expanding $S(X)$ in a Taylor 


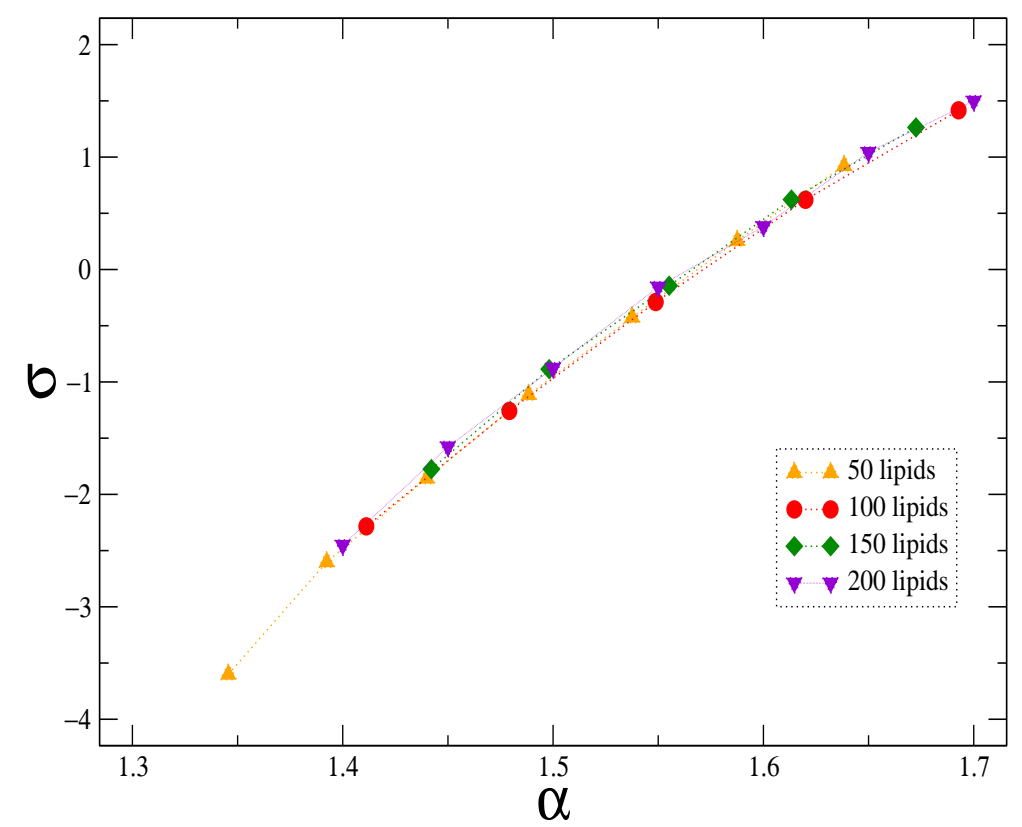

FIG. 2: The surface tension $\sigma$ plotted against the area per molecule $\alpha$ for various values of $N$, the number of molecules constituting the membrane.

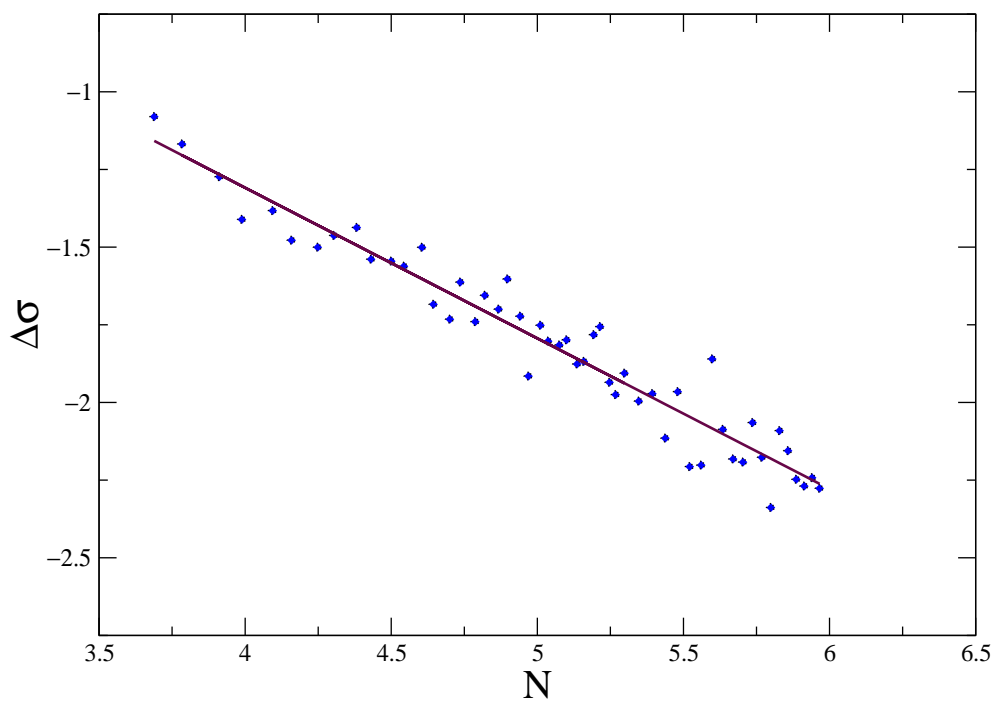

FIG. 3: The mean square fluctuations in the surface tension $\Delta \sigma$ plotted against the number $N$ of molecules constituting the membrane on a log-log plot. The best fit straight line to the data has a slope of .48, very close to the theoretically expected slope of .5 . 
expansion about $X_{0}$, we see that changes in entropy can be approximated by a quadratic form: the Hessian matrix of $S$ with respect to $X$. As first discussed by Einstein, a system in statistical mechanics explores neighboring lower entropy states with probability given by $\exp \Delta S$. Thus there are Gaussian fluctuations of $X$ about $X_{0}$ with a width determined by the eigenvalues of the Hessian matrix. The fluctuations responsible for Brownian motion are exactly of this variety. The equilibrium position of our membrane is a flat surface in the $x-y$ plane with an area determined by the periodic boundary conditions. However, due to thermal fluctuations the membrane explores curved configurations, leading to area as well as surface tension fluctuations. While the fluctuation effects seen and their $N$ dependence is to be expected, they have not so far been experimentally studied. Neither has their theoretical significance for quantum gravity been appreciated. This is what we turn to next.

\section{LEARNING FROM ANALOGY}

Sorkin's suggestion of a fluctuating $\Lambda$ neatly solves the Brobdingnag problem of explaining a small but non zero cosmological constant: $\Lambda$ is as small as it can be, given the uncertainties inherent in quantum gravity. As a bonus, it also automatically solves the "coincidence problem". The measured value for $\Lambda$ today is comparable to $\rho$, the matter density in the Universe. According to the standard picture of a cosmological constant, $\Lambda$ must have been subdominant in the past and is only now starting to dominate the dynamics of the universe. But "why now?", a puzzle known as the coincidence problem. It appears to be a coincidence that we live in the epoch where $\Lambda$ and $\rho$ are of comparable magnitude. In Sorkin's proposal, there is nothing special about our present epoch. $\Lambda \approx \rho$ at all epochs, in four spacetime dimensions. This conclusion of an "everpresent $\Lambda$ " is unaffected by extra dimensions [40] provided only that these are not large extra dimensions, much bigger than the Planck scale. Sorkin's argument implies that the order of magnitude of the fluctuations in $\Lambda$ was larger in the past than it is today. A common reaction from astronomers is that such a theory can be observationally ruled out. This reaction is usually based on the standard notion of cosmological constant which is not a fluctuating quantity. If indeed, Sorkin's idea can be disproved observationally, it would be wonderful, for then, we would have a real confrontation between theory and experiment in quantum gravity. The logic behind Sorkin's suggestion is simple 
enough to be convincing. If a simple chain of logic leads to a conflict with observation (remember Olber's paradox) there is much to be learned since one of the links in the chain must give. However, the idea of a fluctuating cosmological constant is still in its infancy and needs development and precision to make further testable predictions. A number of points remain unclear: What does one mean by a fluctuating cosmological constant? Is it a fixed fluctuation for the whole universe? Does $\Lambda$ depend on cosmic epoch? or cosmic location? Does this not conflict with Einstein's equations which imply a constant $\Lambda$ via the Bianchi identity? What is the role of Einstein's equations? How general is Sorkin's idea? Is it inherently tied to the Causet approach to quantum gravity, or do other approaches also predict a fluctuating $\Lambda$ ?

Let us now learn from the soft matter analogy and address these questions. The first lesson we learn concerns our attitude towards spacetime, gravity and the cosmological constant. The orthodox view of spacetime is that of a smooth manifold. This is a picture that works well over a range of scales. Gravity is normally viewed by relativists as a fundamental theory of pure geometry, and if a cosmological constant is present, $\Lambda$ is viewed as a fixed number whose numerical value is to be determined from experiment. However, borrowing a leaf from condensed matter physics, we see that the geometric description of a membrane as a smooth surface embedded in space is only an emergent picture valid at long (say micron) length scales. At a molecular scale (nanoscale) a membrane is roughened [15] by relative displacements and protrusions of individual molecules jostling for space. The surface tension emerges at low energies as a "chemical potential" describing the energy cost of creating a unit area of membrane. Like any chemical potential, the surface tension can fluctuate for finite systems as we have seen it does. In Gravity, we should by analogy, view GR as an emergent effective description valid at long length scales. There may be a true microscopic theory (similar to the molecular description of membranes) underlying GR, but we do not know what it is and are not here attempting to guess what it is. Whatever the microscopic description is (String Theory, Loop Quantum Gravity, Causets, Non-commutative Geometry or any other candidate), we restrict ourselves to long wavelength phenomena and try to find low energy imprints of Planck scale effects, just as Brownian motion was manifested at micron scales. In this spirit the cosmological constant should be viewed as a "chemical potential" for the creation of spacetime. It is the action cost for creating a unit four volume of spacetime, just as the surface tension is the energy cost for creating a unit area of membrane. Like any chemical potential, the 
cosmological constant too is subject to fluctuations.

What does one mean by a fluctuating cosmological constant? From the membrane analogy, we know that for a flat membrane in its equilibrium configuration, the surface tension is a constant. This follows from the condition for mechanical equilibrium

$$
\nabla_{i} \sigma^{i j}=0
$$

However this equation only holds in the thermally averaged sense. As we saw earlier, a finite lipid membrane has fluctuations about its equilibrium configuration. We would thus expect that the surface tension has local fluctuations which depend on position and have vanishing mean:

$$
<\sigma(x)>=0,<\sigma(x) \sigma\left(x^{\prime}\right)>\neq 0
$$

We would similarly expect in the cosmological context that $\Lambda$ has spacetime dependent fluctuations.

$$
<\Lambda(x)>=0,<\Lambda(x) \Lambda\left(x^{\prime}\right)>\neq 0
$$

We see already from Sorkin's argument (since it can be applied at any epoch) that the fluctuating $\Lambda$ is not a fixed constant for the whole Universe: it does depend on Cosmic time. However, then it would be natural to permit it to have spatial fluctuations as well as temporal ones. We conclude that the $\Lambda$ fluctuations do depend on Cosmic epoch and location.

Einstein's Equations: Einstein's equations imply via the Bianchi identity that $\Lambda$ must be a constant. Yet we have seen that $\Lambda$ has local fluctuations. How does one reconcile the two? The membrane analogy suggests an answer. The condition for mechanical equilibrium (11) is only true on thermal averaging. It is not true as an operator equation. Similarly, in cosmology, we are considering quantum fluctuations about a classical solution (the homogeneous, isotropic Robertson-Walker metric). Quantum fluctuations do not obey the classical Einstein equations. We would still expect Einstein's equations to hold in some average $<G_{\mu \nu}>=<T_{\mu \nu}>$ sense, but there is no reason to expect then to hold as operator equations. Similarly the conservation of matter implied by Einstein's equations classically, $\nabla_{\mu} T^{\mu \nu}=0$ only holds on an average and not as an operator identity. It follows that a fluctuating $\Lambda$ does not have to be a constant in space and time as naively implied by Einstein's equations. 
Sorkin's proposal for a fluctuating $\Lambda$ was made within the context of Causal Sets and Unimodular gravity. How essential are these inputs? Let us consider them in turn.

Causal Sets In Causal Set theory, spacetime is approximated by a sprinkling of discrete points which are Poisson distributed as a result of Local Lorentz invariance. The $\sqrt{N}$ fluctuations have their origin in the Poisson fluctuations of $N$. A Poisson distribution has the property that its variance $\left\langle(N-\langle N\rangle)^{2}>\right.$ is equal to its mean $\langle N\rangle$ for all $N$. Note however, that the lipid membrane system has a distribution of lipids which is certainly not Poisson. There is repulsion between the molecules and their positions are anticorrelated. However, the system still shows $\sqrt{N}$ fluctuations to a good approximation. The $\sqrt{N}$ fluctuations seen in the bilayer are a result of the central limit theorem and the law of large numbers: for large enough $N$, any distribution tends to a Gaussian distribution whose width increases as $\sqrt{N}$. It appears then that Poisson distribution is not essential for $\sqrt{N}$ fluctuations, if $N$ is large enough. In the cosmological context, $\mathcal{N}_{\text {Arch }}$ is comfortably large and we can safely suppose that there will be $\sqrt{\mathcal{N}_{\text {Arch }}}$ fluctuations of the cosmological constant. It appears then that the essential ingredient of Sorkin's argument is discreteness of spacetime, rather than any other aspect of Causet theory (like Local Lorentz invariance).

Unimodular Gravity: What is the role played by Unimodular gravity? To understand this we go back to the membrane system. The surface tension is a thermodynamic potential conjugate to the area of the membrane just as pressure is conjugate to volume. Let us define the Gibbs ensemble partition function $Z_{G}(\sigma)$

$$
Z_{G}(\sigma)=\sum_{\mathcal{C}}\left(\exp -\beta \mathcal{E}_{2}(\mathcal{C})\right)(\exp -\sigma \mathcal{A}(\mathcal{C}))
$$

in which the surface tension is regarded as the control parameter and the area fluctuates. It is also possible to define the "Helmholtz" ensemble partition function

$$
Z_{F}(\mathcal{A})=\sum_{\mathcal{C}} \exp -\beta \mathcal{E}_{2}(\mathcal{C}) \delta(\mathcal{A}-\mathcal{A}(\mathcal{C}))
$$

which describes a system in which the area is held constant and the surface tension fluctuates. The Helmholtz partition function relates to the Gibbs partition function by a Laplace transform:

$$
Z_{G}(\sigma)=\exp -\beta G(\sigma)=\int_{0}^{\infty} d \mathcal{A} e^{-\beta \sigma \mathcal{A}} e^{-\beta F(\mathcal{A})}
$$


For systems at the thermodynamic limit the fluctuations are negligible and the two ensembles are equivalent, but for the finite size systems that we are interested in, there are differences[37]. Which ensemble is appropriate depends on the experimental conditions. If the control parameter is the surface tension then the Gibbs ensemble should be used. One can also envisage more general ensembles in which neither the area nor the surface tension is held constant and both fluctuate. The fluctuations are related by something like an "uncertainty" relation $\Delta \mathcal{A} \Delta \sigma \approx 1$. Indeed this is the situation in the simulations we described in the last section. All we are holding constant in the simulation is the cyclic boundary conditions on the molecules. The area of the membrane and the surface tension show fluctuations of order $\sqrt{N}$ and $1 / \sqrt{N}$ respectively in accord with the "uncertainty" relation.

Using the analogy above we can transcribe the description of a fluid membrane to the gravity context. We can write analogue "partition functions". In both cases (membranes as well as gravity) we are assuming that the microscopic physics provides an ultraviolet cutoff so that the sums in (14,15]17,18) are convergent. The "Gibbs Partition function", which we write formally as

$$
Z_{G}[\Lambda]:=\sum_{\mathcal{H}} \exp i\left(\Lambda * \mathcal{V}+I_{2}\right)
$$

can be viewed as simply one statistical ensemble in which $\Lambda$ is held fixed and its conjugate variable $\mathcal{V}$ fluctuates. One could equally well consider the conjugate Helmholtz ensemble

$$
Z_{F}[\mathcal{V}]:=\sum_{\mathcal{H}} \exp \left(i I_{2}\right) \delta(\mathcal{V}(\mathcal{H})-\mathcal{V})
$$

$I_{2}$ here is the Einstein-Hilbert action (Table 1). If one takes the classical limit and evaluates this path integral by stationary phase one ends up with a theory in which the variations of the metric in the Einstein-Hilbert action are subject to the constraint that the four volume is unchanged. This theory is equivalent to Unimodular Gravity! Thus Unimodular gravity [42, 43] should not be viewed as an exotic theory, but a close cousin of Einstein's general relativity belonging to a different statistical ensemble and related to it by a Legendre transform. Classically the two theories are equivalent. In fact one can conceive of more general ensembles where both $\Lambda$ and $\mathcal{V}$ are allowed to fluctuate. This is no different from having states in quantum mechanics which are neither position nor momentum eigenstates. There are fluctuations in both quantities and these fluctuations satisfy the uncertainty principle. 
It would then seem that the two essential components to Sorkin's idea of a fluctuating cosmological constant are graininess of spacetime and a possibility of fluctuations in $\Lambda$. The first ingredient is present in most approaches to quantum gravity. The second ingredient is easily incorporated by considering a conjugate ensemble. We conclude then that the idea of a fluctuating cosmological constant is not closely tied up with Causets and Unimodular gravity, but is more general. This conclusion is disappointing for Causets, because the cosmological constant then does not favour Causets over competing theories of quantum gravity. On the other hand, the conclusion is a generic prediction of quantum gravity models and so Sorkin's idea gives us a natural resolution of the cosmological constant problem from quantum gravity.

\section{CONCLUSION}

As we remarked earlier, the analogy between the Universe and fluid membranes is a mathematical one and maps physically disparate elements into each other. This sometimes has unexpected benefits. The cosmological constant problem is widely regarded as a difficult one. However, it appears that the analogue problem is almost trivially solved. Free standing fluid membranes have a vanishing surface tension (part a) and have small surface tension fluctuations (part b). While the vanishing surface tension of fluid membranes is regarded as commonplace in the soft matter community, its analogue in cosmology is far from trivial. It is worth spending a few words on why freestanding fluid membranes have vanishing surface tension. The hydrophobic interactions which govern the behaviour of lipid membranes are of high energy $\left(a_{i j}>>T\right)$. Such strong interactions give rise to strong forces that regroup particles (in the form of a bilayer) so that these strong forces are no longer effective. All we see at low energies is the residual effects that remain after these strong forces cancel out. These residual effects are a consequence of imperfect cancellations between the strong forces. Similar physics happens in other systems. Strong electromagnetic forces between protons and electrons are screened out when they form atoms and then interact by weak van der Waal forces. This idea was expressed early and very well by Newton (Opticks): "Now the smallest particles of Matter may cohere by the strongest Attractions, and compose bigger

Particles of weaker Virtue; and many of these may cohere and compose bigger Particles whose Virtue is still weaker, and so on for divers Successions, until the Progression end in the biggest 
particles on which the Operations in Chymistry, and the Colours of natural Bodies depend, and which by cohering compose bodies of a sensible magnitude." Replacing the archaic "Virtue" by its modern equivalents "charge" or "coupling constants" we see that Newton has in mind "emergent behaviour". The idea that physics depends on scale is a prevalent one in condensed matter physics and formalised in the renormalisation group.

Our attitude of viewing the supposedly fundamental theory of general relativity as an emergent description valid at low energies is borrowed from condensed matter physics and is fairly widespread today [21, 46]. In Landau theory, one expands the free energy in powers of decreasing length dimension and discards higher order terms. Landau theory is essentially a low energy theory and has the advantage that we do not need to know the microscopic basis for the theory. The low energy description depends only on general symmetry properties and not on details of the microscopic theory. Our ignorance of microscopics is absorbed into a set of phenomenological constants. For instance altering the values of $a_{i j}$ which describe the forces between atoms will not change the $N$ dependence of $\sigma_{0} / \sqrt{N}$, but could affect the value of $\sigma_{0}$.

For quantum gravity, the microscopic theory is presently a matter of speculation and therefore it is of advantage not to have to commit ourselves to any particular model. Doing effective field theory with gravitation is no harder than doing continuum elasticity, even though matter is fundamentally atomistic. Our ignorance of fundamental physics can be lumped into a few "elastic constants" of which the cosmological constant is one. We must distinguish here between generic predictions which do not depend on the microscopic theory and specific predictions which do. Both have their value: generic predictions give us general quantum gravity insights into cosmology but do not discriminate between different microscopic theories, while specific predictions can hopefully be used to rule out some theories and favour others. For instance, we have seen that the $1 / \sqrt{\mathcal{N}_{\text {Arch }}}$ dependence of the $\Lambda$ fluctuations is generic. But the coefficient which is of order unity may depend on the specific microscopic theory.

We have relied on analogy as a guide to refine Sorkin's idea of a fluctuating cosmological constant. Like all analogies, our analogy between GR and membranes has its limitations (see table 2). 


\section{Limitations of Analogy}

\begin{tabular}{ll} 
Membranes & Universe \\
dimension two & dimension four \\
Euclidean geometries & Lorentzian geometries \\
Positive $\sigma$ minimises area & Positive $\lambda$ causes accelerated expansion \\
No Causal Structure & Causal Structure \\
Boundary value problem & Initial value problem \\
Ambient Space and Extrinsic geometry & Purely Intrinsic geometry \\
Exponentially damped sum over configurations & Oscillatory phase sum over histories \\
Non-Poissonian distribution of molecules & Poissonian distribution of Causet elements \\
\hline
\end{tabular}

TABLE II: Table 2 shows some limitations of the analogy between General Relativity and fluid membranes

Obvious differences are those of dimension (four versus two) and signature (Lorentzian versus Euclidean). As a result of these differences, a positive $\Lambda$ tends to accelerate the expansion of the Universe, whereas a positive $\sigma$ tends to contract a membrane! The Action of spacetime depends on purely intrinsic geometry, while the membrane energy depends on both intrinsic and extrinsic geometry. There is no analogue in membranes of the Causal structure of spacetime which was a prime motivating force in Sorkin's approach. The membrane problem is Euclidean and best formulated as a boundary value problem, while in GR because of the Lorentzian signature, an initial value problem is more natural. Despite these differences, the fluctuations expected in Sorkin's approach appear in membranes and lead us to believe that Sorkin's idea is truly more general than its original context. With all its limitations, the analogy is a useful one and suggests future work in several directions.

Sorkin's idea of a fluctuating lambda is still incompletely explored. We feel it is a compelling idea which should be pursued to its logical conclusion and confronted with observational evidence. Barrow [6] has argued that observational Cosmic Microwave Background Radiation (CMBR) evidence rules out the idea of "Everpresent $\Lambda$ " in the form it was developed by Ahmed et al [1]. But this avatar of Sorkin's idea[41] holds fast to some components of Einstein's equations, while giving up others. Barrow's criticism too has recourse to Einstein's equations. It would be nice to develop Sorkin's idea in its full generality using stochastic differential equations to replace Einstein's equa- 
tions and then try to rule it out convincingly by using CMBR obervations and improved supernova data which may become available in the future [20]

One observable quantity which could be used to confront theory with experiment is the spectrum of fluctuation. The power spectrum of surface tension fluctuations is easily explored using computer techniques as well as analytical calculations[28]. This information is contained in the Fourier transform of the two point correlation functions $<\sigma(x) \sigma\left(x^{\prime}\right)>$. Since $\Lambda$ has spacetime dependent fluctuations, one could expect that its spectrum of fluctuations [48] can also be calculated from $<\Lambda(x) \Lambda\left(x^{\prime}\right)>$. Since the spectrum of metric fluctuations is well constrained by CMBR observations, this could provide a confrontation between theory and experiment. There may be aspects of the spectrum that are specific and depend on the microscopic theory, in which case the observations may be used to rule out candidate quantum theories of gravitation. On the other hand if the predictions are generic and independent of the microscopic theory, a conflict with observations would cast a shadow of doubt on the whole venture of quantum gravity.

The $\Lambda$ fluctuations may be accessible by numerical quantum gravity, a developing area in which there is considerable current interest. There is much work [2] that explores quantum gravity on a computer using dynamical triangulations and spin networks. Just as we are able to see fluctuating $\sigma$ in fluid membranes by numerical experiments, it should be possible to see fluctuating $\Lambda$ in numerical quantum gravity. Apart from the magnitude of the effect, it should also be possible to work out the spectrum of fluctuations, which is an imporant observable quantity. Our work suggests that by using computer methods like dynamical triangulations it should be possible to see effects of fluctuating $\Lambda$ in every approach to quantum gravity. It is also perhaps true that the simulation methods are more highly developed in condensed matter physics than in numerical quantum gravity. Techniques analogous to DPD which improve the efficiency of computer runs may be a useful import into numerical quantum gravity.

The smooth picture we have of spacetime in GR may yield in quantum gravity to some discrete microscopic structure. Such views about geometry and spacetime were expressed very early by Riemann and Einstein (see [16]). Riemann in his inaugural lecture on geometry discusses continuous manifolds as well as discrete ones, evidently regarding the latter as more natural. "..Their quantitative comparison happens for discrete manifolds through counting, for continuous one through measurement." Einstein, in a letter to to Dällenbach, (1916) says "...But you have 
correctly grasped the drawback that the continuum brings. If the molecular view of matter is the appropriate one, i.e., if a part of the Universe is to be represented by a finite number of moving points, then the continuum of the present theory contains too great a manifold of possibilities. I also believe that this too great is responsible for the fact that our present means of description miscarry with the quantum theory. The problem seems to me how one can formulate statements about a discontinuum without calling upon a continuum..."

It may be as Riemann and Einstein anticipated that the spacetime continuum emerges only in the low energy approximation that is accessible to us through our feeble probes. Our best particle accelerators today are but coarse instruments by Planck scale standards, reminiscent of optical microscopes in nanophysics. The underlying discreteness of spacetime resists our best efforts, just as atoms escaped detection for two millenia from the time of Democritus. If they are numerous enough, discrete points (atoms of matter or of spacetime) can appear continuous as the stars on the Milky Way. To get at the underlying discreteness, our best chance may be to look at emergent fluctuation effects. This is the main lesson we have learned from Einstein's work on Brownian motion and the main point of this paper. We hope that the bridge we have made between quantum gravitational physics and membrane physics will trigger further work in experiments, simulations and theory in both areas.

Acknowledgements: It is a pleasure to thank Maddalena Venturoli for her simulation programs for the surface tension of lipid membranes, Abhishek Choudhury for many discussions on this work, Irina Pushkina for discussions on numerical quantum gravity, David Finkelstein for a conversation about the analogy and Joe Henson, Satya Majumdar, John Ipsen, Michael Fisher, Bei-Lok Hu and Ted Jacobson for their comments.

[1] Ahmed M, Dodelson S, Greene P B and Sorkin R 2004 Phys. Rev. D 69, 103523.

[2] Ambjorn J, Anagnostopoulos K N, Loll R and Pushkina I 2009 Nucl. Phys. B807, 251-264.

[3] Amelino-Camelia G, Ellis J R, Mavromatos N E, Nanopoulos D V and Sarkar S 1998 Nature 393, 763-765.

[4] Archimedes Circa 250 BC http://en.wikipedia.org/wiki/The_Sand_Reckoner. 
[5] Barcelo, C. Liberati S and Visser M 2005 Living Reviews in Relativity 8, 12.

[6] Barrow J D 2007 Phys. Rev. D75, 067301.

[7] Bombelli L, Lee J, Meyer D and Sorkin R D 1987 Phys. Rev. Lett. 59(5), 521-524.

[8] Bowick M J, Chandar L, Schiff E A and Srivastava A M 1994 Science 263, 943.

[9] Copeland E J, Sami M and Tsujikawa S 2006 Int. J. Mod. Phys. D15, 1753-1936.

[10] Dirac P A M 1974 Proc. Roy. Soc A, 338(1615), 439-446.

[11] Dowker F, Henson J and Sorkin R D 2004 Mod. Phys. Lett. A19, 1829-1840.

[12] et al R C 2003 Journal of Physics A: Mathematical and General 36(23), 6281.

URL: http://stacks.iop.org/0305-4470/36/i=23/a=301

[13] et al R C 2005 Journal of Physics A: Mathematical and General 38(41), 8841.

URL: http://stacks.iop.org/0305-4470/38/i=41/a=002

[14] Frenkel D and Smit B 2002 Understanding Molecular Simulations Academic Press, Elsevier USA.

[15] Goetz R, Gompper G and Lipowsky R 1999 Phys. Rev. Lett. 82(1), 221-224.

[16] Gomberoff A and Marolf D 2005 Lectures on Quantum Gravity Springer New York.

[17] Groot R and Rabone K 2001 Biophysical Journal 81, 725.

[18] Henriksen J and Ipsen J 2004 EuroPhysics Journal E 14, 149-167.

[19] Hoogerbrugge P and Koelman J 1992 Europhys. lett. 19, 155-160.

[20] Howell D A et al. 2009 arxiv/astro-ph/0903.1086.

[21] Hu B L 2009 eprint arXiv/gr-qc/0903.0878.

[22] Kranenburg M, Venturoli M and Smit B 2003 Phys. Rev. E 67(6), 060901.

[23] Kwok R and Evans E 1981 Biophysical Journal 35, 637.

[24] Landau L and Lifshitz E 2000 Statistical Physics Pergammon Press.

[25] Nelson D, Weinberg S and Piran T 2004 Statistical Mechanics of Membranes and Surfaces .

[26] Nielsen H B and Olesen P 1973 Nucl. Phys. B 61, 45.

[27] Padmanabhan T 2008 Gen. Rel. Grav. 40, 529.

[28] Peliti L and Leibler S 1985 Phys. Rev. Lett. 54, 1690.

[29] Perlmutter S et al. 1999 Astrophys. J. 517, 565-586.

[30] Philpott L, Dowker F and Sorkin R D 2008.

[31] Riess A G et al. 1998 Astron. J. 116, 1009-1038. 
[32] Safran S A 1994 Statistical Thermodynamics of Surfaces, Interfaces and Membranes .

[33] Sahni V, Krasinski A and Zeldovich Y B 2008 Gen. Rel. Grav. 40, 1557-1591.

[34] Samuel J and Sinha S 2006 Phys. Rev. Lett. 97, 161302.

[35] Schofield P and Henderson J 1982 Proc. Roy. Soc. A 379, 231-246.

[36] Shillcock J C and Lipowsky R 2002 Journal Of Chemical Physics 117, 5048.

[37] Sinha S and Samuel J 2005 Phys. Rev. E 71(2), 021104.

URL: http://link.aps.org/abstract/PRE/v71/e021104

[38] Sorkin R 1991 First Steps with Causal Sets in General Relativity and Gravitational Physics Proceedings of the Ninth Italian Conference on GR and Gravitational Physics, Capri, Italy, September 1990 ( R. Ciani, R de Ritis, M. Francaviglia, G. Marmo, C. Rubano and P. Scudellaro, eds.), pp 68-70, World Scientific, Singapore.

[39] Sorkin R D 1997 Int. J. Theor. Phys. 36, 2759.

[40] Sorkin R D 2005 Braz. J. Phys. 35, 280-283.

[41] Sorkin R D 2007 AIP Conf. Proc. 957, 142-153.

[42] Unruh W G 1989 Phys. Rev. D 40(4), 1048-1052.

[43] Unruh W G and Wald R M 1989 Phys. Rev. D 40(8), 2598-2614.

[44] Vachaspati T and Vilenkin A 1984 Physical Review D 30, 2036.

[45] Vilenkin A and Shellard E P S 1994 Cosmic Strings and other topological defects.

[46] Volovik G 2003 The Universe in a Helium Droplet Clarendon Press, Oxford.

[47] Volovik G E 2004 JETP Lett. 80, 465-468.

[48] Volovik G E 2008 arXiv/gr-qc/0801.2714.

[49] Wood-Vasey W M et al. 2007 Astrophys. J. 666, 694-715. 